unterstützen und zu bestärken «. Dieses Urteil, das Rechtsanwalt Groenewold (vermutlich in richtiger Einschätzung des BGH) und die Bundesanwaltschaft rechtskräftig werden ließen, bedeutet eine Preisgabe des durch definierte Tatbestandsmerkmale bestimmten Strafprozesses.

Jürgen Seifert

\title{
Urteil des Oberverwaltungsgerichts Münster vom 23. August 1977
}

In dem Verwaltungsrechtsstreit des [ . . ]

- als Kläger - [...]

gegen

den Oberstadtdirektor der Stadt Düsseldorf - Rechtsamt -, [ . . ]

- als Beklagten - [...]

hat der VIII. Senat $[\ldots]$

durch

den Vorsitzenden Richter am Oberverwaltungsgericht Dr. Maué,

den Richter am Oberverwaltungsgericht Frege,

die Richterin am Verwaltungsgericht Dr. Schauer,

den ehrenamtlichen Richter Jubr, Bankangestellter,

den ehrenamtlichen Richter Koppers, Gewerkschaftssekretär,

auf die Berufung des Klägers gegen das Urteil des Verwaltungsgerichts Düsseldorf

vom 9. Juli 1975

für Recht erkannt:

Die Berufung wird zurückgewiesen. [...]

Tatbestand

Der Kläger besuchte in der Zeit von August 1971 bis Ende 1973 das Wilhelm-Heinrich-Riehl-Kolleg in Düsseldorf, eine staatlich anerkannte Ersatzschule des Zweiten Bildungsweges zur Erlangung der Hochschulreife. Das Kolleg ist eine Tagesschule mit Pflichtunterricht vorwiegend in den Vormittagsstunden. Für diese Ausbildung erhielt der Kläger vom Beklagten Ausbildungsförderung, die sich im Bewilligungszeitraum vom I. August 1972 bis 31. Juli 1973 auf 427,- DM monatlich belief.

In der Zeit vom 24. Januar bis I. Februar 1973 einschließlich blieb der größte Teil der Studierenden des Kollegs, unter ihnen der Kläger, dem weiterhin angebotenen Unterricht fern, um gegen die Streichung eines monatlichen Zuschusses zum Mensaessen zu demonstrieren. Wegen dieses Unterrichtsboykotts machte der Beklagte gegenüber dem Kläger durch Bescheid vom 28. Februar 1973 einen Rückzahlungsanspruch in Höhe von I25,40 DM geltend, da dieser Betrag nach seinen Berechnungen auf die versäumten neun Schultage entfiel. Zugleich teilte er mit, daß er diesen Betrag in zwei Raten von dem Förderungsbetrag des Klägers einbehalten werde. Den Widerspruch des Klägers gegen diesen Bescheid wies das Landesamt für Ausbildungsförderung Nordrhein-Westfalen durch Widerspruchsbescheid vom 5. Juli 1973 zurück $[\ldots]$.

$[\ldots]$

Durch Urteil vom 9. Juli 1975 hat das Verwaltungsgericht Düsseldorf die Klage abgewiesen. [...] 
[...] Die Berufung hat keinen Erfolg.

Das Verwaltungsgericht hat die Klage zu Recht abgewiesen. Der angefochtene Rückforderungsbescheid ist nicht gemäß $\$ II $33_{3}$ Abs. I Satz I VwGO aufzuheben; denn er ist rechtmäßig und verletzt den Kläger nicht in seinen Rechten.

Anzuwenden ist das Bundesausbildungsförderungsgesetz in der Urfassung vom 26. August 197 (BGBl. I S. I409) - BAföG -.

Der Beklagte hat zu Recht einen Betrag in Höhe von I25,40 DM von dem Kläger zurückgefordert. Gemäß $\ 20$ Abs. 2 BAföG ist der Förderungsbetrag für den Kalendermonat oder den Teil eines Kalendermonats zurückzuzahlen, in dem der Auszubildende die Ausbildung aus einem von ihm zu vertretenden Grund unterbrochen hat. Ob die Ausbildung im Sinne des $\$ 20$ Abs. 2 BAföG »unterbrochen« worden ist, unterliegt in vollem Umfang der verwaltungsrechtlichen Nachprüfung, da es sich um einen unbestimmten Rechtsbegriff handelt. Der Kläger hat die Kollegausbildung, für die er Ausbildungsförderung erhielt, unterbrochen; denn er hat in der Zeit von Mittwoch, dem 24. Januar bis Donnerstag, dem I. Februar 1973 einschließlich wegen Beteiligung an einem Unterrichtsboykott nicht an den Unterrichtsveranstaltungen des Wilhelm-Heinrich-Riehl-Kollegs in Düsseldorf teilgenommen. Die Unterrichtsveranstaltungen des Kollegs haben, wie der Kläger selbst einräumt, während des Unterrichtsboykotts weiterhin stattgefunden, da nicht alle Klassen und aus den verbliebenen Klassen nicht alle Studierenden am Unterrichtsboykott teilgenommen haben. Hat ein Auszubildender an einem Kolleg während der Dauer von insgesamt neun aufeinanderfolgenden Tagen an dem planmäßig vorgesehenen und tatsächlich angebotenen Unterricht nicht teilgenommen, so rechtfertigt dies die Feststellung, daß er seine Ausbildung für diesen Zeitraum im Sinne des $\ 20$ Abs. 2 BAföG unterbrochen hat.

Um eine bloße Vernachlässigung der Ausbildung handelt es sich nicht. Eine bloße Vernachlässigung der Ausbildung, die keine Unterbrechung der Ausbildung darstellt, kann im Falle des Fernbleibens von Unterrichtsveranstaltungen gegeben sein, wenn - wie vielfach im Hochschulbereich - nach der Ausbildungs- und Prüfungsordnung eine Pflicht zur Anwesenheit bei den belegten Vorlesungen nicht besteht, der Auszubildende aber während des betreffenden Zeitraums an Seminaren, Ubungen und Praktika teilnimmt.

(Bundesverwaltungsgericht (BVerwG), Urteil vom 17. Oktober 1974-V C 4 I.73-, Zeitschrift für das gesamte Familienrecht (FamRZ) 1975, II 3 = Fürsorgerechtliche Entscheidungen der Verwaltungs- und Sozialgerichte (FEVS) 23, I 39; Bayerischer Verwaltungsgerichtshof, Urteil vom 19. Juli 1973 - Nr. I 90 VII 72 -, Die Offentliche Verwaltung (DÖV) 1974, 68.)

Der Kläger absolvierte jedoch keine Hochschulausbildung, in der der Besuch aller angebotenen Lehrveranstaltungen nicht obligatorisch ist, sondern eine Kollegausbildung. Für die Kollegausbildung ist die Pflicht zur Teilnahme am Unterricht in der Prüfungsordnung verbindlich vorgeschrieben. Gemäß dem Runderlaß des Kultusministers des Landes Nordrhein-Westfalen vom 27. Januar 1971 (Gemeinsames Amtsblatt - GABl. - S. I72) - RE - ist die »Vorläufige Reifeprüfungsordnung für das Staatliche Institut zur Erlangung der Hochschulreife in Oberhausen « - RPO - vom I0. August 1955 (Amtsblatt des Kultusministers - ABIKM - S. I25), für alle anderen Kollegs für verbindlich erklärt worden. Nach $\$ 4 \mathrm{Abs}$. I RPO können sich zur Reifeprüfung jene ordentlichen Studierenden der normalen Kurse melden, die am Unterricht ... teilgenommen haben. Diese in der RPO normierte Pflicht der Studierenden an einem Kolleg, am Unterricht teilzunehmen, hat auch in $\ 7$ Abs. 
I der Institutsordnung des Wilhelm-Heinrich-Riehl-Kollegs vom 25. Juli 1967- IO - ihren Niederschlag gefunden, in dem es heißt:

"Die Studierenden sind zum regelmäßigen und pünktlichen Besuch des Unterrichts und der vom Kollegleiter festgesetzten Kollegveranstaltungen verpflichtet.« [...]

$\mathrm{Da}$ die Pflicht zur Teilnahme am Unterricht in der RPO und auch in der IO normiert ist, hat der Kläger durch sein Fernbleiben vom Unterricht seine Ausbildung selbst dann unterbrochen, wenn er - wie er behauptet - sich durch häusliches Studium seiner Ausbildung gewidmet hat. Eine förderungsfähige Kollegausbildung kann nach dem Studienplan nur durch den Besuch des Unterrichts und nicht allein durch häusliche Studien absolviert werden.

Falls der Kläger, wie er behauptet, in der Zeit des Unterrichtsboykotts regelmäßig an den Studierenden-Vollversammlungen teilgenommen hat, die nach seinen Angaben täglich mit Ausnahme des 25. Januar 1973 und des I. Februar 1973 während des gesamten Vormittags und teilweise auch noch am Nachmittag stattgefunden haben, steht dieser Umstand nicht der Feststellung entgegen, daß der Kläger in dieser Zeit seine Ausbildung unterbrochen hat. Die Ausbildung im Sinne des $\ 20$ Abs. 2 BAföG wird nur dann fortgesetzt und nicht unterbrochen, wenn der Auszubildende an den für ihn obligatorischen Ausbildungsveranstaltungen teilnimmt. Ausbildungsveranstaltungen waren die Studierenden-Vollversammlungen jedenfalls nicht, da sie kein für das Ausbildungsziel relevantes Fachwissen vermittelten. $\mathrm{Ob}$ die Studierenden-Vollversammlungen als "Schulveranstaltungen" im Sinne des Runderlasses des Kultusministers vom 16 . Oktober 1968 zur Schülermitverwaltung (ABl. KM S. 304) - RESMV - anzusehen sind, kann dahingestellt bleiben. Nicht alle Schulveranstaltungen sind gleichzeitig Ausbildungsveranstaltungen. Zu den Schulveranstaltungen, die keine Ausbildungsveranstaltungen sind, zählen außer den Veranstaltungen im Rahmen der Schülermitverwaltung zum Beispiel Ausflüge, Besuche unterhaltender Veranstaltungen, Schulfest, Schulgottesdienst usw. Wer nur an derartigen Schulveranstaltungen teilnimmt, ohne die im Ausbildungsplan vorgesehenen Ausbildungsveranstaltungen zu besuchen, unterbricht seine Ausbildung $[\ldots]$.

Der Kläger kann sich auch nicht mit Erfolg darauf berufen, daß jedenfalls eine oder zwei der Studierenden-Vollversammlungen vom Schulleiter genehmigt und folglich als Schulveranstaltung anzusehen seien, und daß er durch die Teilnahme daran jedenfalls nicht länger als 3 Tage ununterbrochen den sonstigen Schulveranstaltungen, d. h. dem Unterricht, ferngeblieben sei. Selbst wenn man diese Prämissen unterstellt, hätte der Kläger seine Ausbildung unterbrochen. Zwar ist in Tz. 20.2.2 Satz Ia der Allgemeinen Vorschrift zum BAföG vom 25. August I976 (GMBl. S. 386) - BAföG - VwV - geregelt, daß als Unterbrechung im Sinne des $\$ 20$ Abs. 2 BAföG bei Besuch eines Kollegs eine Unterbrechung von mehr als 3 aufeinanderfolgenden Unterrichts- und Vorlesungstagen gilt. [...]

Eine konsequente Anwendung der Tz. 20.2.2 Satz Ia BAföG - VwV bzw. BAföG VwV-E hätte zur Folge, daß ein Auszubildender nach dreitägigem Unterrichtsboykott nur einen Tag lang an dem planmäßig vorgesehenen Unterricht voll teilzunehmen und seine Hausarbeiten durchzuführen brauchte und sodann drei Tage lang die Ausbildung boykottieren könnte, ohne daß sein Verhalten als Unterbrechung der Ausbildung zu werten wäre. Eine derartige Konsequenz steht mit Sinn und Zweck des $\ 20$ Abs. 2 BAföG nicht im Einklang. Nach der Begründung zu $\ 20$ Abs. 2 des Regierungsentwurfs des BAföG (Bundestags-Drucksache VI/I975) verdeutlicht diese Bestimmung, »daß nur für - und darum nur während - der Ausbildung die Lebensgrundlage des Auszubildenden durch Ausbildungsförderung sichergestellt wird «. Ein Auszubildender, der in einem gewissen zeitlichen Zusammenhang des 
öfteren einzelne Tage lang dem Unterricht fernbleibt, zwischendurch aber wieder am Unterricht teilnimmt, unterbricht seine Ausbildung ebenso wie derjenige, der einmal ununterbrochen mehrere Tage lang den Unterricht versäumt. [.. .]

Bei Anwendung dieser Grundsätze ist festzustellen, daß der Kläger auch dann seine Ausbildung unterbrochen hätte, wenn der Schulleiter einzelne der StudierendenVollversammlungen als Schulveranstaltungen genehmigt hätte und der zur gleichen Zeit vorgesehene Unterricht ausgefallen wäre, selbst wenn zwischen diesen Veranstaltungen weniger als drei Tage gelegen hätten. $\mathrm{Da}$ der Kläger den sonstigen während der Zeit des Unterrichtsboykotts in allen Fächern angebotenen Unterrichtsveranstaltungen ferngeblieben ist, hat er sich nicht ganz überwiegend seiner Ausbildung gewidmet, sondern sich in beträchtlichem Umfang während der normalen Unterrichtszeit in unterrichtsfremder Weise beschäftigt [...].

Der Kläger hat die Ausbildung auch aus einem von ihm zu vertretenden Grund unterbrochen. Er ist vorsätzlich den Unterrichtsveranstaltungen ferngeblieben. Auf einen triftigen Hinderungsgrund kann er sich nicht berufen. Ob er ein Recht zum Unterrichtsboykott hatte, dessen Grundlagen im übrigen nicht ersichtlich sind, ist unerheblich; denn für die wirtschaftlichen Folgen der Ausübung demokratischer Rechte hat der Auszubildende ebenso einzustehen wie der junge Erwerbstätige. (Vgl. Begründung zu $\ 20$ Abs. 2 des Regierungsentwurfs des BAföG, a. a. O.)

$[\ldots]$

Dr. Mané

Frege

Dr. Schaner

Az.: VIII A $1572 / 75$

7 K $2125 / 73$ Düsseldorf

\section{Urteil des Verwaltungsgerichts Gelsenkirchen vom I 3. Oktober 1977}

In dem Verwaltungsrechtsstreit

der Frau $[\ldots]$

Klägerin, [...]

gegen

den Oberstadtdirektor der Stadt Dortmund - Amt für Ausbildungsförderung -, [...]

Beklagten, $[\ldots]$

hat die 2. Kammer des Verwaltungsgerichts Gelsenkirchen aufgrund der mündlichen Verhandlung in der Sitzung

vom ${ }_{13}$. Oktober 1977

durch

den Vizepräsidenten des Verwaltungsgerichts Dr. Wagemann,

den Richter am Verwaltungsgericht Schwitzke,

den Richter am Verwaltungsgericht Frielinghaus,

den ehrenamtlichen Richter Bernhard Gosmann, und

den ehrenamtlichen Richter Karl Pieper

für Recht erkannt:

I. Der Bescheid des Beklagten vom I5. Dezember 1975 und der Widerspruchsbescheid des Landesamtes für Ausbildungsförderung Nordrhein-Westfalen vom 6. Februar 1976 werden aufgehoben [...] 